\title{
Nomogram predicting cesarean delivery undergoing induction of labor among high- risk nulliparous women at term: a retrospective study
}

Hang Zhou, Ning Gu, Yan Yang, Zhiqun Wang, Yali Hu and Yimin Dai

\begin{abstract}
Background: Our aim was to create and validate a nomogram predicting cesarean delivery after induction of labor among nulliparous women at term.

Methods: Data were obtained from medical records from Nanjing Drum Tower Hospital. Nulliparous women with singleton pregnancies undergoing induction of labor at term were involved. A total of 2950 patients from Jan. 2014 to Dec. 2015 were served as derivation cohort. A nomogram was constructed by multivariate logistic regression using maternal, fetal and pregnancy characteristics. The predictive accuracy and discriminative ability of the nomogram were internal validated by 1000-bootstrap resampling, followed by external validation of a new dataset from Jan. 2016 to Dec. 2016.

Results: Logistic regression revealed nine predictors of cesarean delivery, including maternal height, age, uterine height, abdominal circumference, estimated fetal weight, indications for induction of labor, initial cervical consistency, cervical effacement and station. Nomogram was well calibrated and had an AUC of 0.73 ( $95 \%$ confidence interval [Cl], 0.70-0.75) after bootstrap resampling for internal validation. The AUC in external validation reached 0.67 , which was significantly higher than that of three models published previously $(P<0.05)$.
\end{abstract}

Conclusions: This validated nomogram, constructed by variables that were obtained form medical records, can help estimate risk of cesarean delivery before induction of labor.

Keywords: Induction of labor, Cesarean delivery, Nomogram, Internal validation, External validation

\section{Background}

Induction of labor is one of the most frequently used methods to initiate labor. In the United States, more than $22 \%$ pregnancies undergo induction of labor and almost one third of inductions will end in cesarean delivery [1].

\footnotetext{
*Correspondence: nj_daiyimin@126.com
}

Department of Obstetrics and Gynecology, Nanjing Drum Tower Hospital, Nanjing University Medical School, 321 Zhongshan Road, Nanjing 210008, Jiangsu, China
The status of cervix has been recognized as one of the most important factors affecting the mode of delivery, and an unfavorable Bishop score $(\leq 5)$ is the predominant risk factor of failed induction [2, 3]. Other studies have proposed that, several maternal and fetal characteristics, such as maternal age, parity, maternal height, body mass index, gestational age and fetal position, also affect the success rate of labor induction [4-8].

It has been widely assumed that induction of labor increases the risk of cesarean delivery. Previous studies have reported that cesarean delivery rates varied from 
22.6 to $50 \%$ among nulliparous after induction of labor in different institutes, most of which included patients for both medical and non-medical indications [9-13]. However, one randomized trial has demonstrated that elective induction can decrease risk of cesarean delivery among low-risk nulliparous [14]. For high-risk nulliparous, indications for labor induction, such as diabetes mellitus and hypertension, have been reported to associate with cesarean delivery [10].

However, there is little knowledge about how to evaluate the risk so far, since these factors are seldom used in a comprehensive fashion. Therefore, it is reasonable to develop and validate a nomogram of cesarean delivery for nulliparous undergoing induction of labor, by combination of maternal, fetal and pregnancy characteristics.

\section{Materials and methods}

The protocol of the retrospective study was approved by the Ethic Review Committee of Nanjing Drum Tower Hospital (reference number 2020-027-01, date of approval 2020-02-25). Written informed consent was obtained from all women enrolled in the program. All nulliparous with singleton, term, cephalic pregnancies who underwent induction of labor from Jan. 2014 to Dec. 2016 at Nanjing Drum Tower Hospital were enrolled.

Data on maternal and neonatal characteristic were abstracted from medical record. These data were doublechecked by two obstetricians. Women with cervical dilation $\geq 3 \mathrm{~cm}$ were excluded since they might have been in spontaneous labor and were misclassified as induction of labor. Besides, women with missing data were also excluded for further data analysis. Factors enrolled for analysis were 1) maternal demographic characteristics, including maternal age, maternal height, maternal weight at delivery, uterine height and abdominal circumference; 2) medical indications for induction of labor, including premature rupture of membrane, late term, diabetes mellitus (gestational diabetes and pregestational diabetes), hypertensive disorder of pregnancy, liver dysfunction, fetal growth restriction and oligohydramnios; 3) obstetric conditions, including gestational age, fetal position, cervical examination at admission (cervical dilation, effacement, position, consistency and station), Bishop score; and 4) neonatal characteristics, including estimated fetal weigh, neonatal weight, neonatal sex. Results of PROBAAT trial and further meta-analysis has demonstrated that cesarean delivery rate was similar between Foley catheter group and prostaglandin group [15]. Besides, the original purpose of the retrospective study was to provide a user-friendly tool for both doctors and patients. The procedures of induction might be too professional and make patients confused. Therefore, methods of induction were not included in the study.
Gestational age was determined by the last menstrual period and confirmed by ultrasound examination. Timing to start induction was mainly based American College of Obstetricians and Gynecologists (ACOG) committee opinion "Medically indicated late-preterm and earlyterm deliveries" published in April 2013 [16].

The admission exam was the full 5 component Bishop score (cervical dilation, effacement, position, consistency and station). A Bishop score of $<6$ was considered unfavorable. All women with intact membrane whose Bishop score $<6$ received at least 1 method for cervical ripening: Foley catheter, vaginal misoprostol $25 \mu$ g every $4 \mathrm{~h}$ or vaginal dinoprostone (Propess). The standard application of Foley catheter has been established by our group previously and chosen as the first choice for cervical ripening for women without vaginal infection [16]. The choice of vaginal misoprostol or dinoprostone (Propess) was mainly based on provider preference. Artificial rupture of membrane was considered once the Bishop score $\geq 6$.

Women who had successful vaginal delivery after induction of labor were classified as successful induction of labor; those who ended with cesarean delivery for any reason after induction of labor were classified as failed induction of labor. The model was constructed using derivation cohort from Jan. 2014 to Dec. 2015, which has been used to analyze cesarean delivery rate by 10 -Group Classification System [17]. The dataset of Group 2a (nulliparous, single cephalic, $\geq 37$ week, induced labor) had detailed variables for evaluation and perfectly fitted the target population in our research. Women with same inclusion criteria form Jan. 2016 to Dec. 2016 were enrolled as validation cohort. Continuous variables were presented as mean and standard deviation or median and interquartile range, where appropriate. Categorical variables were presented as frequencies and percentages. Student $t$ test and Chi square test were used for continuous and categorical date in univariate analysis, respectively. All variables with a $P<0.05$ in univariate analysis were then included in a logistic regression model. Covariates were removed in a stepwise fashion until all covariates in the final value had a $P<0.05$. A nomogram was created based on coefficients weighted by the logistic regression model in R. The nomogram was internal and external validated by discrimination and calibration. Discrimination was assessed by receiver-operative characteristics (ROC) analysis using 1000 bootstrap resampling, and Calibration curve was graphically assessed by plotting the observed rates against the nomogram-predicted probabilities.

To compare the nomogram with other existing prediction model, we searched Medline between 1987 and 2020 on prediction model for induction of labor. The search strategy consisted of keywords of "induction of labor" 
and "prediction model". Studies were selected in a twostage process. Firstly, we went through title and abstract of all citations. Secondly, we obtained full reports published in English which established mathematical models to predict cesarean delivery after induction of labor, with sufficient detail to calculate the probability of cesarean delivery using our own data. Studies on both nulliparous and multiparous were also included. Area under the curve (AUC) for ROC analysis between the nomogram and models finally included were compared.

\section{Results}

During 2014 to 2015, a total of 11,006 deliveries occurred at our hospital, of which 2961 met inclusion criteria. Due to incomplete data, 11 pregnancies were excluded. Therefore, 2950 pregnancies were finally enrolled as derivation cohort. A total of 1935 pregnancies admitted in 2016 were enrolled as validation cohort.

Table 1 showed the antepartum characteristics of pregnancies in derivation and validation cohort. The cesarean delivery rate slightly in increased from $13.2 \%$ in derivation cohort to $16.4 \%$ in validation cohort. PROM, consisted of over $40 \%$ of study population, remained the first indication for induction of labor, followed by late term, which consisted of $25 \%$ of the population. The derivation cohort and validation cohort shared similar characteristics in both maternal and fetal features.

Maternal, neonatal and obstetric characteristics were compared between women who delivered vaginally and women who delivered by cesarean delivery by univariate analysis (Table 2). Detailed information about indications for induction of labor among women in derivation cohort was presented in Table 3.

In univariate analysis, the following variables had a $P<0.05$ and were considered in logistic regression modeling: maternal age, weight, height, uterine height, abdominal circumference, gestational age, estimated fetal weight, initial cervical dilation, initial station, initial cervical effacement, initial cervical consistency and indications for induction of labor. After stepwise logistic regression analysis, independent risk factors associated with cesarean delivery after induction of labor were shown in Table 4. The following 9 variables remained significantly associated with cesarean delivery: maternal age, height, uterine height, abdominal circumference, estimated fetal weight, initial station, initial cervical effacement, initial cervical consistency and indications for induction of labor. For every 1-year increase in maternal age, there was a $9 \%$ increase in the odds of cesarean delivery (odds ratio [OR] 1.09, 95\% confidence interval [CI] 1.05-1.13). Decreased maternal height was associated with increased probability of cesarean delivery (OR 0.91, 95\% CI 0.88-0.93). A $1 \mathrm{~cm}$ increase in uterine height,
$1 \mathrm{~cm}$ increase in abdominal circumference and $100 \mathrm{~g}$ increase in estimated fetal weight were associated with 6.0\% (OR 1.07, 95\%CI 1.00-1.13), 5.0\% (OR 1.05, 95\%CI $1.03-1.08$ ) and $6.0 \%$ (OR 1.06, 95\% CI 1.01-1.12) increase in the odds of cesarean delivery, respectively. Three components of Bishop score, including initial station, initial cervical effacement and initial cervical consistency were associated with risk of cesarean delivery. Initial cervical effacement levels $60-70 \%, 40-50 \%$ and $0-30 \%$ were associated with increased risk of cesarean delivery (OR 1.79, 95\%CI 0.97-3.31; OR 2.19 95\%CI 1.14-4.21; OR 9.23, $95 \%$ CI 1.26-67.56). Hypertensive disorder of pregnancy was one of the most important risk factors, since it had over a 2-fold risk of cesarean delivery (OR 2.38, 95\%CI 1.38-4.13), followed by late term, which associated with $58.0 \%$ increase in the odds of cesarean delivery (OR 1.58, 95\%CI 1.20-2.08).

The nomogram was created based on coefficients of parameters enrolled in the final logistic regression (Fig. 1). The receiver operating characteristic curve for the nomogram achieved an area under the curve (AUC) of 0.73 (95\%CI, 0.70-0.75) for internal validation and 0.67 (95\%CI, 0.64-0.702) for external validation after 1000 bootstrap resampling. The sensitivity and specificity of the nomogram reached 0.63 and 0.71 , respectively. The calibration curve for both derivation and validation cohort revealed good agreement between predicted risk of cesarean delivery after induction of labor by the nomogram and actual observation, as shown in Fig. 2A and B.

The initial literature review revealed 271 hit. After reading titles and abstracts, 18 articles on cesarean delivery after induction of labor were identified. Fifteen literatures were excluded due to various reasons. Details on literature selection were presented in Fig. S1.

The remaining 3 models were finally included in our study [18-20]. The probability of cesarean delivery after induction of labor for each patient was calculated according to the mathematical equation published in article. The areas under the curve were $0.68,0.66$ and 0.64 for Robert model, Antonio model and Gordon model, respectively, which were significantly lower than AUC of our nomogram in derivation dataset (Fig. 3A). Meanwhile, similar results were found when applying validation dataset to these models (Fig. 3B).

\section{Discussion}

In this single-institutional retrospective cohort of high-risk nulliparous women who underwent induction of labor, we found that maternal age, height, uterine height, abdominal circumference, estimated fetal weight, initial station, initial cervical effacement and initial cervical consistency were independent risk factors for cesarean delivery. Besides, indications for 
Table 1 Antepartum characteristics of nulliparous undergoing induction of labor at term

\begin{tabular}{|c|c|c|c|}
\hline Characteristic & $\begin{array}{l}\text { Training Cohort } \\
N=2950\end{array}$ & $\begin{array}{l}\text { Validation Cohort } \\
N=1935\end{array}$ & $P$ \\
\hline Maternal age (y) & $28.4 \pm 3.1$ & $28.6 \pm 3.0$ & 0.016 \\
\hline Weight (kg) & $71.0 \pm 9.3$ & $71.2 \pm 9.1$ & 0.431 \\
\hline Height (cm) & $162.0 \pm 4.5$ & $161.9 \pm 4.6$ & 0.742 \\
\hline Uterine height (cm) & $35.5 \pm 2.2$ & $35.4 \pm 2.2$ & 0.032 \\
\hline Abdominal circumference $(\mathrm{cm})$ & $101.3 \pm 6.3$ & $101.2 \pm 6.1$ & 0.780 \\
\hline Gestational age (wk) & $39(39-41)$ & $40(39-41)$ & 0.007 \\
\hline Fetal sex & & & 0.585 \\
\hline Male & $1542(52.3)$ & $996(51.5)$ & \\
\hline Female & $1408(47.7)$ & $939(48.5)$ & \\
\hline Estimated fetal weight (g) & $3500(3200-3600)$ & $3500(3200-3600)$ & 0.270 \\
\hline Initial cervical dilation (cm) & & & 0.002 \\
\hline 0 (0 points) & 2880 (97.6) & $1913(98.9)$ & \\
\hline 1-2 (1 points) & $70(2.4)$ & $22(1.1)$ & \\
\hline Initial station & & & 0.106 \\
\hline-3 (0 points) & $962(65.5)$ & $583(30.1)$ & \\
\hline-2 (1 points) & $1932(32.6)$ & $1322(68.3)$ & \\
\hline-1 or 0 (2 points) & $56(1.9)$ & $30(1.6)$ & \\
\hline + 1 (3 points) & - & - & \\
\hline Initial cervical effacement (\%) & & & $<0.001$ \\
\hline 0-30 (0 points) & $5(0.2)$ & $2(0.1)$ & \\
\hline 40-50 (1 points) & $520(17.6)$ & $256(13.2)$ & \\
\hline 60-70 (2 points) & $2205(74.7)$ & $1492(77.1)$ & \\
\hline$\geq 80$ (3 points) & $220(7.5)$ & $185(9.6)$ & \\
\hline Initial cervical position & & & $<0.001$ \\
\hline Posterior (0 points) & $1015(34.4)$ & $570(29.5)$ & \\
\hline Mid position (1 points) & $1875(63.6)$ & $1352(69.9)$ & \\
\hline Anterior (2 points) & $60(2.0)$ & $13(0.7)$ & \\
\hline Initial cervical consistency & & & $<0.001$ \\
\hline Firm (0 points) & $44(1.5)$ & $17(0.9)$ & \\
\hline Medium (1 points) & $1495(50.7)$ & $1102(57.0)$ & \\
\hline Soft (2 points) & $1411(47.8)$ & $816(42.2)$ & \\
\hline Bishop Score & $5(4-5)$ & $5(4-5)$ & 0.621 \\
\hline$<6$ & $2185(74.1)$ & $1421(73.4)$ & \\
\hline$\geq 6$ & $765(25.9)$ & $514(26.6)$ & \\
\hline Indications for induction of labor & & & $<0.001$ \\
\hline PROM & $1319(44.8)$ & $941(48.6)$ & \\
\hline Late term (41 weeks or greater) & $779(26.4)$ & $522(27.0)$ & \\
\hline Diabetes mellitus & $472(16.0)$ & $167(8.6)$ & \\
\hline Hypertensive disorder of pregnancy & $81(2.7)$ & $108(5.6)$ & \\
\hline Others & $299(10.1)$ & 197(10.2) & \\
\hline Cesarean delivery & $392(13.3)$ & $317(16.4)$ & \\
\hline
\end{tabular}

Data are presented as mean \pm standard derivation, number (\%) or range PROM Premature rupture of membrane

induction of labor also independently affected the rate of cesarean delivery. Using these factors, a nomogram was developed and validated to calculate the likelihood of cesarean delivery, which achieved acceptable AUC of
0.73 for internal validation and 0.67 for external validation. Further analysis revealed that the nomogram showed better discriminative ability than three models published previously. 
Table 2 Univariate analysis of antepartum characteristics of nulliparous in train cohort

\begin{tabular}{|c|c|c|c|}
\hline Characteristics & $\begin{array}{l}\text { Vaginal Delivery } \\
N=2558\end{array}$ & $\begin{array}{l}\text { Cesarean Delivery } \\
N=392\end{array}$ & $P$ \\
\hline Maternal age (y) & $28.2 \pm 3.1$ & $29.4 \pm 3.4$ & $<0.001$ \\
\hline Weight (kg) & $70.7 \pm 9.1$ & $73.1 \pm 10.4$ & $<0.001$ \\
\hline Height (cm) & $162.1 \pm 4.5$ & $160.5 \pm 4.4$ & $<0.001$ \\
\hline Uterine height (cm) & $35.4 \pm 2.2$ & $36.2 \pm 2.4$ & $<0.001$ \\
\hline Abdominal circumference $(\mathrm{cm})$ & $100.9 \pm 6.1$ & $103.6 \pm 6.8$ & $<0.001$ \\
\hline Gestational age (wk) & $39(39-40)$ & $40(39-41)$ & 0.003 \\
\hline Estimated fetal weight (g) & $3500(3200-3600)$ & $3500(3300-3700)$ & $<0.001$ \\
\hline Initial cervical dilation (cm) & & & 0.025 \\
\hline 0 (0 points) & $2491(97.4)$ & $389(99.2)$ & \\
\hline 1-2 (1 points) & $67(2.6)$ & $3(0.8)$ & \\
\hline Initial station & & & $<0.001$ \\
\hline-3 (0 points) & $783(30.6)$ & $179(45.7)$ & \\
\hline-2 (1 points) & $1723(67.4)$ & $209(53.3)$ & \\
\hline-1 or 0 (2 points) & $52(2.0)$ & $4(1.0)$ & \\
\hline \multicolumn{4}{|l|}{+1 (3 points) } \\
\hline Initial cervical effacement (\%) & & & $<0.001$ \\
\hline 0-30 (0 points) & $2(0.1)$ & $3(0.8)$ & \\
\hline 40-50 (1 points) & $430(16.8)$ & $90(23.0)$ & \\
\hline 60-70 (2 points) & $1918(75.0)$ & $287(73.2)$ & \\
\hline$\geq 80$ (3 points) & $208(8.1)$ & $12(3.1)$ & \\
\hline Initial cervical position & & & 0.336 \\
\hline Posterior (0 points) & $893(34.9)$ & $122(31.1)$ & \\
\hline Mid position (1 points) & $1613(63.1)$ & $262(66.8)$ & \\
\hline Anterior (2 points) & $52(2.0)$ & $8(2.0)$ & \\
\hline Initial cervical consistency & & & $<0.001$ \\
\hline Firm (0 points) & $35(1.4)$ & $9(2.3)$ & \\
\hline Medium (1 points) & $1256(49.1)$ & $239(61.0)$ & \\
\hline Soft (2 points) & $1266(49.5)$ & $144(36.7)$ & \\
\hline Bishop Score & & & $<0.001$ \\
\hline$<6$ & $1853(72.4)$ & $332(84.7)$ & \\
\hline$\geq 6$ & 705 (27.6) & $60(15.3)$ & \\
\hline
\end{tabular}

Data are presented as mean \pm standard derivation, number (\%) or range

$P$ values were calculated by Student $t$ test or Chi square test, as appropriate

The risk factors enrolled in the nomogram have been previously reported in literatures. In consistent with previous studies, maternal age and height were associated with cesarean delivery [10, 20-23]. Meanwhile, medical indications for induction of labor, such as PROM, late term, diabetes mellitus and hypertensive disorder of pregnancy were also shown to be independently affected rate cesarean delivery $[10,23]$. In our research, uterine height, abdominal circumference and estimated fetal weight were modifiable risk factor, influencing probability of cesarean delivery. Traditionally, Bishop score has been used as the standard evaluation for induction planning. However, not all components were related to cesarean delivery $[10,23]$. Cervical dilation, a favorable factor for vaginal delivery, was not recognized as a risk factor in our study. This was likely due to the fact that only a few patients were enrolled with dilated cervix. Meanwhile, cervical position was not associated with cesarean delivery after adjusting for confounders in the large cohort.

Efforts have been made to assess the risk of cesarean delivery among different populations and AUC of these models ranged from 0.68-0.79. Gordon et al. established a prediction model for cesarean delivery after labor induction in nulliparous by four risk factors, including maternal age, height, gestational age and fetal sex [18]. 
Table 3 Indications for induction of labor among nulliparous women

\begin{tabular}{|c|c|c|}
\hline Indications & $\begin{array}{l}\text { Vaginal Delivery } \\
N=2558\end{array}$ & $\begin{array}{l}\text { Cesarean Delivery } \\
N=392\end{array}$ \\
\hline PROM & $1182(46.2)$ & $137(34.9)$ \\
\hline Late term (41 weeks or greater) & $647(25.3)$ & $132(33.7)$ \\
\hline Diabetes mellitus & $408(15.9)$ & $64(16.3)$ \\
\hline $\begin{array}{l}\text { Hypertensive disorder of } \\
\text { pregnancy }\end{array}$ & $58(2.3)$ & $23(5.9)$ \\
\hline Others & $263(10.3)$ & $36(9.2)$ \\
\hline Liver dysfunction & $61(23.2)$ & $9(25.0)$ \\
\hline Fetal growth restriction & $30(11.4)$ & $3(8.3)$ \\
\hline Oligohydramnios & $172(65.4)$ & $24(66.7)$ \\
\hline
\end{tabular}

Data are presented as number (\%)

PROM Premature rupture of membrane

The AUC were 0.68 for internal validation and 0.67 for external validation, which seemed lower than our nomogram. Meanwhile, models published by Antonio Hernández-Martínez and Robert. M Rossi revealed better AUC of 0.77 (95\%CI 0.73-0.80) and 0.79 (95\%CI 0.764-0.802) for both nulliparous and multiparous women after induction of labor, respectively $[19,20]$. The differences might result from population composition, since multiparous women were more likely to experience successful vaginal delivery. Therefore, we chose to emphasize on nulliparous women as efforts to reduce primary cesarean delivery regarding its contribution to cesarean delivery rate.

To further compare the discriminative ability of our nomogram and three models mentioned previously, we performed a validation process, using both derivation dataset and validation dataset. AUC of our nomogram reached 0.73 for internal validation and 0.67 for external validation, which showed better accuracy. Therefore, our nomogram might be more specialized for nulliparous in Chinese population.

Our study aimed to establish a nomogram predicting probability of cesarean delivery after induction of labor. The nomogram achieved clinical useful prediction of cesarean delivery by basic characteristics. However, we should aware that the nomogram is constructed to help with patient consultation instead of making clinical decision directly. For example, a nulliparous woman of 30 years (26 points) with hypertensive disorder (22

Table 4 Independent risk factors for cesarean delivery among nulliparous women undergoing induction of labor at term

\begin{tabular}{|c|c|c|c|c|}
\hline Characteristics & Unadjusted OR (95\%Cl) & $P$ & Adjusted OR $(95 \% \mathrm{Cl})$ & $P$ \\
\hline Maternal age (y) & $1.11(1.07-1.14)$ & $<0.001$ & $1.09(1.05-1.13)$ & $<0.001$ \\
\hline Height $(\mathrm{cm})$ & $0.92(0.90-0.95)$ & $<0.001$ & $0.91(0.88-0.93)$ & $<0.001$ \\
\hline Uterine height (cm) & $1.17(1.11-1.22)$ & $<0.001$ & $1.07(1.00-1.13)$ & 0.038 \\
\hline Abdominal circumference $(\mathrm{cm})$ & $1.07(1.05-1.08)$ & $<0.001$ & $1.05(1.03-1.08)$ & $<0.001$ \\
\hline Estimated fetal weight $(100 \mathrm{~g})$ & $1.14(1.09-1.19)$ & $<0.001$ & $1.06(1.01-1.12)$ & 0.013 \\
\hline Initial station & & $<0.001$ & & $<0.001$ \\
\hline-1 or 0 (2 points) & Ref & & Ref & \\
\hline-2 (1 points) & $1.58(0.57-4.40)$ & & $1.26(0.44-3.62)$ & \\
\hline-3 (0 points) & $2.97(1.06-8.32)$ & & $1.96(0.68-5.68)$ & \\
\hline Initial cervical effacement (\%) & & $<0.001$ & & 0.046 \\
\hline$\geq 80$ (3 points) & Ref & & Ref & \\
\hline $60-70$ (2 points) & $2.59(1.43-4.70)$ & & $1.79(0.97-3.31)$ & \\
\hline 40-50 (1 points) & $3.63(1.94-6.78)$ & & $2.19(1.14-4.21)$ & \\
\hline $0-30$ (0 points) & $26.00(3.96-170.64)$ & & $9.23(1.26-67.56)$ & \\
\hline Initial cervical consistency & & $<0.001$ & & 0.006 \\
\hline Soft (2 points) & Ref & & Ref & \\
\hline Firm (0 points) & $2.26(1.07-4.80)$ & & $2.33(1.06-5.11)$ & \\
\hline Medium (1 points) & $1.67(1.34-2.09)$ & & $1.45(1.14-1.83)$ & \\
\hline Indications for induction of labor & & $<0.001$ & & $<0.001$ \\
\hline PROM & Ref & & Ref & \\
\hline Late term (41 weeks or greater) & $1.76(1.36-2.28)$ & & $1.58(1.20-2.08)$ & \\
\hline Diabetes mellitus & $1.35(0.99-1.86)$ & & $1.03(0.73-1.44)$ & \\
\hline Hypertensive disorder of pregnancy & $3.42(2.05-5.72)$ & & $2.38(1.38-4.13)$ & \\
\hline Others & $1.18(0.80-1.75)$ & & $1.22(0.81-1.85)$ & \\
\hline
\end{tabular}

PROM Premature rupture of membrane, OR Odds ratio 


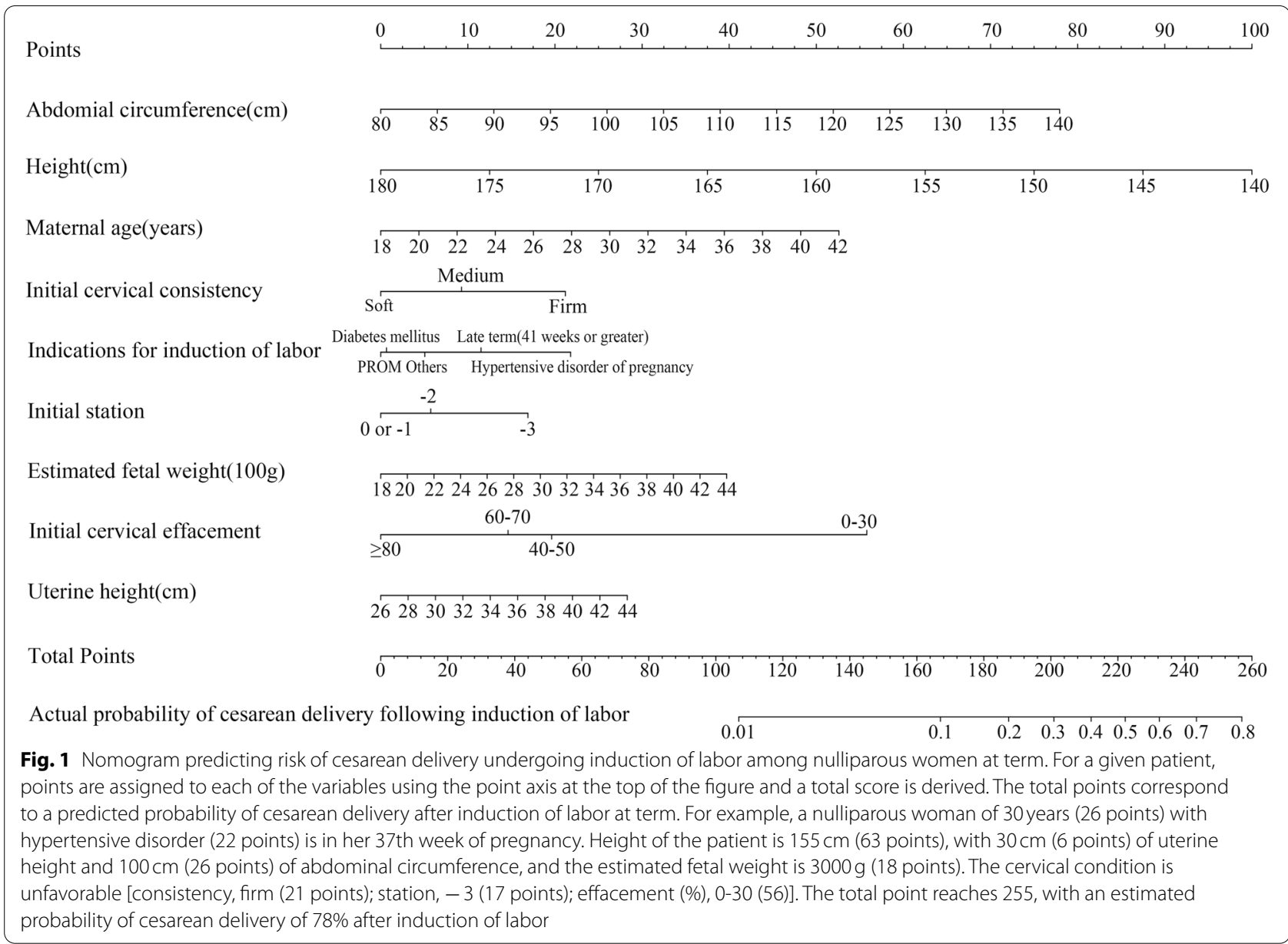

points) is in her 37th week of pregnancy. Height of the patient is $155 \mathrm{~cm}$ (63 points), with $30 \mathrm{~cm}$ (6 points) of uterine height and $100 \mathrm{~cm}$ (26 points) of abdominal circumference, and the estimated fetal weight is $3000 \mathrm{~g}$ (18 points). The cervical condition is unfavorable [consistency, firm (21 points); station, -3 (17 points); effacement (\%), 0-30 (56)]. The total point reaches 255 , with an estimated probability of cesarean delivery of $78 \%$ after induction of labor. The chance of cesarean delivery is extremely high and the women could have chosen to expect a spontaneous labor if there is no strong indication for induction.

Because induction of labor will continue to be one important method for pregnancies at risk of maternal and neonatal morbidity, future studies should focus on patients with high risk of cesarean delivery to choose the safest option.

Our results were robust for several reasons. First, we used large, well-described datasets from one retrospective study on changes of cesarean delivery rate by
10-Group Classification System to establish the nomogram. It provided data with high quality. Secondly, variables enrolled in the nomogram could be easily measured antepartumly. Hence, the nomogram will be easy and inexpensive to daily clinical application. Importantly, both internal and external validation of the nomogram was carried out to ensure its reproducibility in more generalized population. Meanwhile, better discriminative ability of the nomogram was revealed by comparison with three existing models.

The main limitation of the study was the retrospective design. Potential predictors, like pre-pregnancy weight, were not taken into account, because information of these characteristics was not documented in our medical records. Second, the application of the nomogram should be limited to patients who met inclusion criteria of the research. For example, patients with elective induction of labor should not be consulted by the nomogram, since the nomogram was constructed from nulliparous with medical indications 

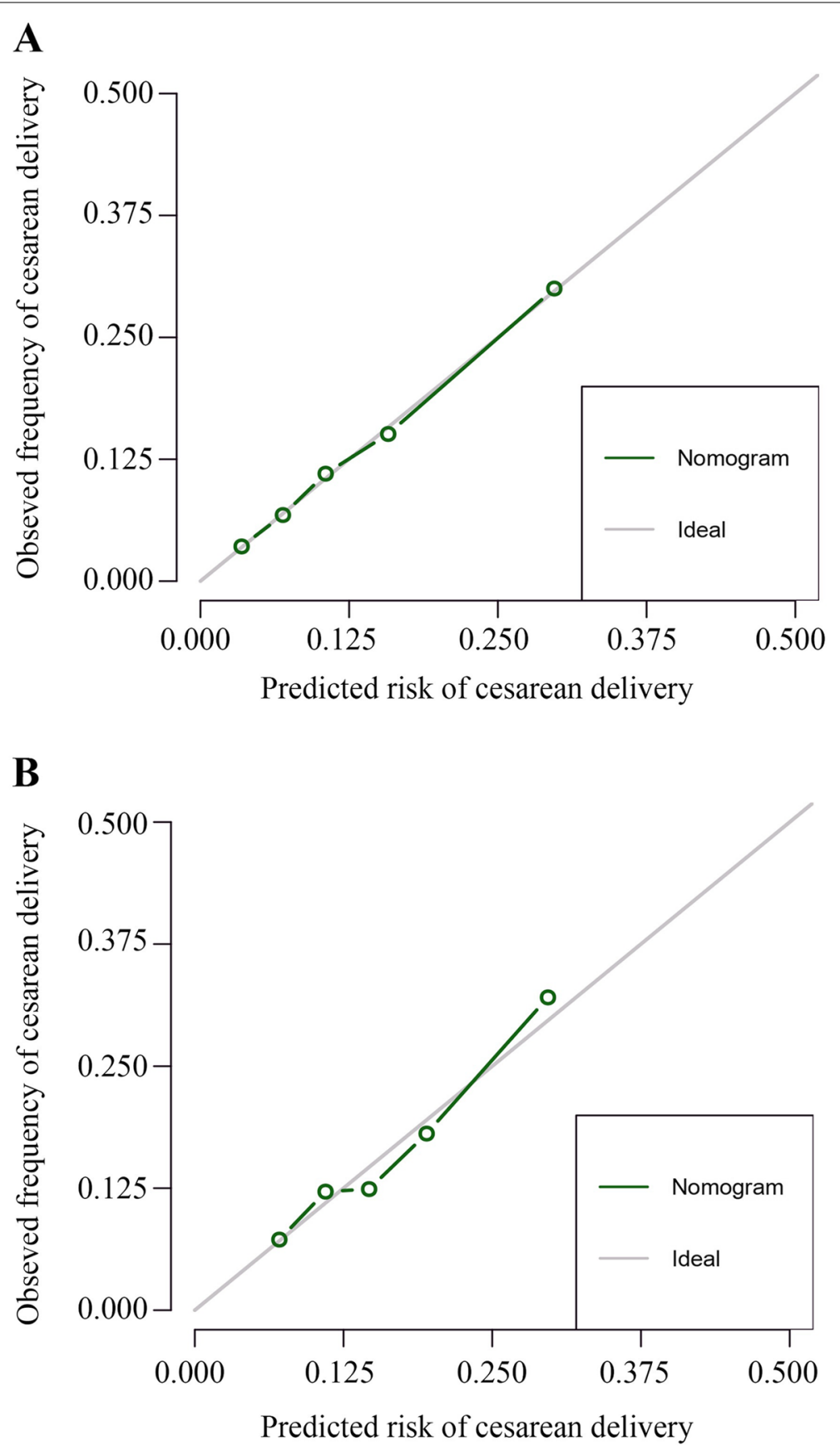

Fig. 2 Calibration curve for the final multivariable model depicted in the nomogram. The calibration curve for predicting risk of cesarean delivery among nulliparous at term after induction of labor in $\mathbf{A}$ derivation cohort and $\mathbf{B}$ validation cohort. The grey line indicates the ideal reference line where the predicted probabilities estimated from the model would match the observed proportion of cesarean delivery. Nomogram-predicted probability is plotted on the $x$-axis, and the actual observation proportion is plotted on the $y$-axis

for induction of labor. Third, external validation of data from other institute should be carried out due to the single institute nature of our study. Besides, relationships between different characteristics and indications of cesarean delivery should be further evaluated to improve the nomogram. 
A

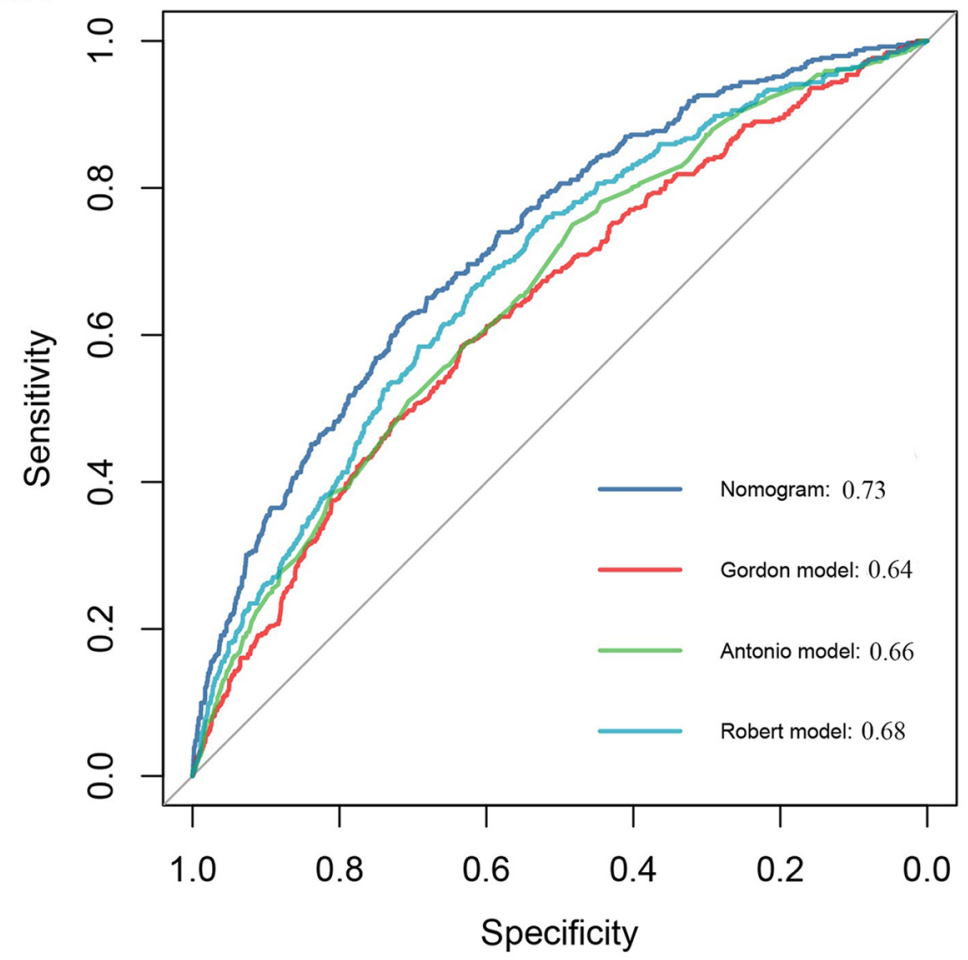

B

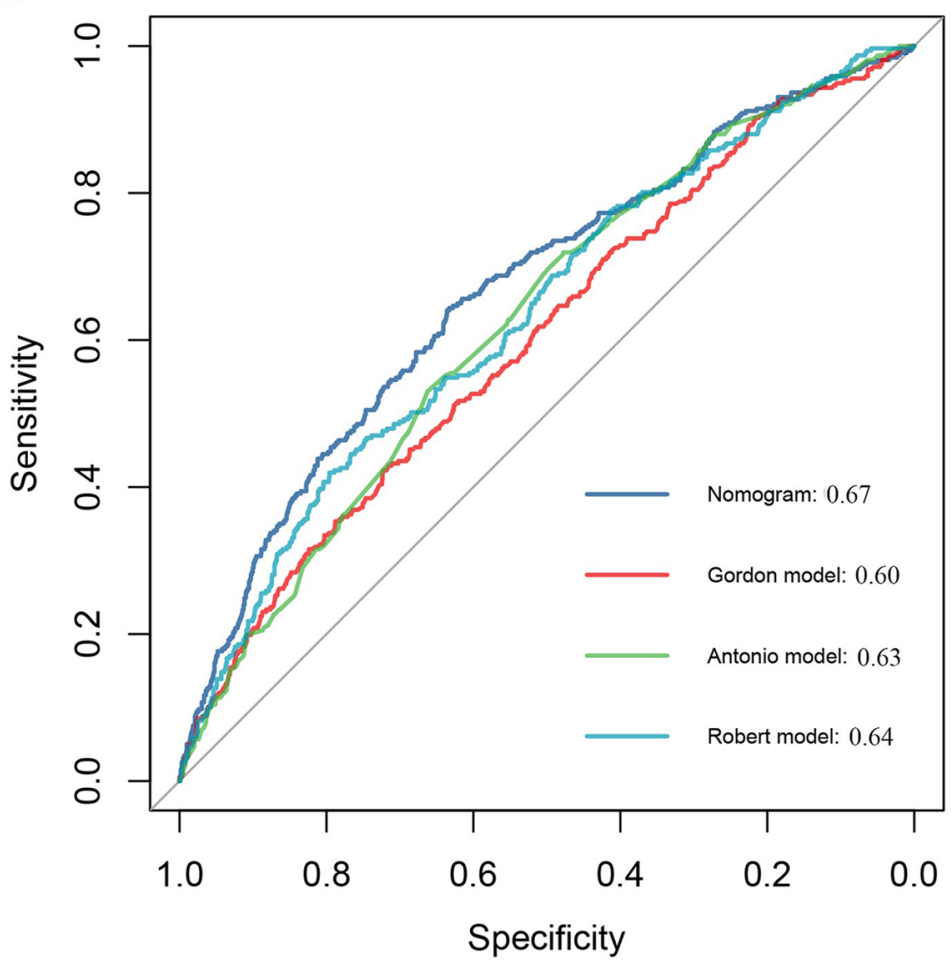

Fig. 3 Receiving operating curve (ROC) of the nomogram and existing models. ROC of the nomogram and existing models for derivation cohort (A) and validation cohort (B). AUC of the nomogram reaches 0.73 in derivation cohort, which is significantly higher than three existing models $(P<0.05)$. Meanwhile, AUC the nomogram reaches 0.67 in validation cohort, which is also significantly higher than three existing models $(P<0.05)$ 


\section{Conclusion}

We constructed a nomogram for cesarean delivery after induction of labor in nulliparous women. The nomogram performed well in both internal and external validation. By providing probability of cesarean delivery after induction of labor, our nomogram would help to consult with delivery mode before undergo of this obstetric procedure.

\section{Abbreviations}

PROM: Premature rupture of membrane; ROC: Receiver-operative characteristics; AUC: Area under the curve; Cl: Confidence interval; OR: Odds ratio; SD: Standard deviation; wk: Week.

\section{Supplementary Information}

The online version contains supplementary material available at https://doi. org/10.1186/s12884-022-04386-8.

Additional file 1: Supplementary Figure 1. Flowchart of literature review of existing models for labor induction. Flowchart of literature review of existing models for labor induction in a two-step process. A total of 253 articles are excluded after reading titles and abstracts, and 15 articels are excluded after going though full text.

\section{Acknowledgments}

Not applicable.

\section{Authors' contributions}

$H Z, N G$ and $Y D$ designed the study. NG, YY and ZW collected the data for analysis. $\mathrm{HZ}, \mathrm{YH}, \mathrm{ZW}$ and $\mathrm{YD}$ analyzed and interpreted the data. $\mathrm{HZ}, \mathrm{NG}$ and $\mathrm{YH}$ conducted the literature search. HZ, NG wrote the first draft of the article. All authors discussed the results and commented on the manuscript. All authors have read and approved the manuscript.

\section{Funding}

This study was endorsed by Jiangsu Provincial Key Medical Center (YXZXB2016004).

\section{Availability of data and materials}

The datasets used and/or analyzed during the current study are available from the corresponding author on reasonable request.

\section{Declarations}

\section{Ethics approval and consent to participate}

All methods of the study were carried out in accordance with Declaration of Helsinki and the protocol of the retrospective study was approved by the Ethic Review Committee of Nanjing Drum Tower Hospital (reference number 2020027-01, date of approval 2020-02-25). Written informed consent was obtained from all women enrolled in the study.

\section{Consent for publication}

Not applicable.

\section{Competing interests}

The authors declare that they have no competing interests.

Received: 24 August 2021 Accepted: 5 January 2022

Published online: 21 January 2022

\section{References}

1. ACOG Practice Bulletin No. 107. Induction of labor. Obstet Gynecol. 2009;114(2 Pt 1):386-97.
2. Baacke KA, Edwards RK. Preinduction cervical assessment. Clin Obstet Gynecol. 2006;49(3):564-72.

3. Vrouenraets FP, Roumen FJ, Dehing CJ, van den Akker ES, Aarts MJ, Scheve EJ. Bishop score and risk of cesarean delivery after induction of labor in nulliparous women. Obstet Gynecol. 2005;105(4):690-7.

4. Kozinszky Z, Orvos H, Zoboki T, Katona M, Wayda K, Pal A, et al. Risk factors for cesarean section of primiparous women aged over 35 years. Acta Obstet Gynecol Scand. 2002;81(4):313-6.

5. Rane SM, Guirgis RR, Higgins B, Nicolaides KH. The value of ultrasound in the prediction of successful induction of labor. Ultrasound Obstet Gynecol. 2004;24(5):538-49.

6. Usha Kiran TS, Hemmadi S, Bethel J, Evans J. Outcome of pregnancy in a woman with an increased body mass index. BJOG. 2005;112(6):768-72.

7. Crane JM, Delaney T, Butt KD, Bennett KA, Hutchens D, Young DC. Predictors of successful labor induction with oral or vaginal misoprostol. J Matern Fetal Neonatal Med. 2004;15(5):319-23.

8. Fitzpatrick M, McQuillan K, O'Herlihy C. Influence of persistent occiput posterior position on delivery outcome. Obstet Gynecol. 2001;98(6):1027-31.

9. Bueno B, San-Frutos L, Salazar F, Perez-Medina T, Engels V, Archilla B, et al. Variables that predict the success of labor induction. Acta Obstet Gynecol Scand. 2005;84(11):1093-7.

10. Tolcher MC, Holbert MR, Weaver AL, McGree ME, Olson JE, El-Nashar SA, et al. Predicting cesarean delivery after induction of labor among nulliparous women at term. Obstet Gynecol. 2015;126(5):1059-68.

11. Gokturk U, Cavkaytar S, Danisman N. Can measurement of cervical length, fetal head position and posterior cervical angle be an alternative method to Bishop score in the prediction of successful labor induction? J Matern Fetal Neonatal Med. 2015;28(11):1360-5.

12. Bahadori F, Ayatollahi H, Naghavi-Behzad M, Khalkhali H, Naseri Z. Predicting factors on cervical ripening and response to induction in women pregnant over 37 weeks. Med Ultrason. 2013;15(3):191-8.

13. de Hundt M, Vlemmix F, Bais JM, de Groot CJ, Mol BW, Kok M. Risk factors for cesarean section and instrumental vaginal delivery after successful external cephalic version. J Matern Fetal Neonatal Med. 2016;29(12):2005-7.

14. Grobman WA, Rice MM, Reddy UM, Tita ATN, Silver RM, Mallett G, et al. Labor induction versus expectant management in low-risk nulliparous women. N Engl J Med. 2018;379(6):513-23.

15. Jozwiak M, Oude Rengerink K, Benthem M, van Beek E, Dijksterhuis MG, de Graaf IM, et al. Foley catheter versus vaginal prostaglandin E2 gel for induction of labour at term (PROBAAT trial): an open-label, randomised controlled trial. Lancet. 2011;378(9809):2095-103.

16. ACOG committee opinion no. 560. Medically indicated late-preterm and early-term deliveries. Obstet Gynecol. 2013;121(4):908-10.

17. Zhang Y, Gu N, Wang Z, Zheng M, Hu Y, Dai Y. Use of the 10-group classification system to analyze how the population control policy change in China has affected cesarean delivery. Int J Gynaecol Obstet. 2017;138(2):158-63.

18. Smith GC, Dellens M, White IR, Pell JP. Combined logistic and Bayesian modeling of cesarean section risk. Am J Obstet Gynecol. 2004;191(6):2029-34.

19. Hernandez-Martinez A, Pascual-Pedreno Al, Bano-Garnes AB, MeleroJimenez MR, Tenias-Burillo JM, Molina-Alarcon M. Predictive model for risk of cesarean section in pregnant women after induction of labor. Arch Gynecol Obstet. 2016;293(3):529-38.

20. Rossi RM, Requarth E, Warshak CR, Dufendach KR, Hall ES, DeFranco EA. Risk calculator to predict cesarean delivery among women undergoing induction of labor. Obstet Gynecol. 2020;135(3):559-68.

21. Schuit E, Kwee A, Westerhuis ME, Van Dessel HJ, Graziosi GC, Van Lith JM, et al. A clinical prediction model to assess the risk of operative delivery. BJOG. 2012;119(8):915-23.

22. Levine LD, Downes KL, Parry S, Elovitz MA, Sammel MD, Srinivas SK. A validated calculator to estimate risk of cesarean after an induction of labor with an unfavorable cervix. Am J Obstet Gynecol. 2018;218(2):254 e251-7.

23. Kawakita T, Reddy UM, Huang CC, Auguste TC, Bauer D, Overcash RT. Predicting vaginal delivery in nulliparous women undergoing induction of labor at term. Am J Perinatol. 2018;35(7):660-8.

\section{Publisher's Note}

Springer Nature remains neutral with regard to jurisdictional claims in published maps and institutional affiliations. 